\title{
Down-regulation of XIAP by AEG35156 in paediatric tumour cells induces apoptosis and sensitises cells to cytotoxic agents
}

\author{
SARAH V. HOLT ${ }^{1}$, KAREN E. BROOKES ${ }^{1}$, CAROLINE DIVE $^{1,2}$ and GUY W.J. MAKIN ${ }^{1,2}$ \\ ${ }^{1}$ Clinical and Experimental Pharmacology, Paterson Institute for Cancer Research and, ${ }^{2}$ School of Cancer and Enabling \\ Sciences, Manchester Cancer Research Centre, The University of Manchester, Wilmslow Road, Manchester M20 4BX, UK
}

Received November 11, 2010; Accepted December 28, 2010

DOI: $10.3892 /$ or.2011.1167

\begin{abstract}
Resistance to conventional chemotherapy is a major problem in several paediatric tumours. One explanation for this is that tumour cells are unable to engage apoptosis after cytotoxic drug-induced damage. Inhibitor of apoptosis proteins (IAPs) function by inhibiting both effector (9) and initiator (3 and 7) caspases. Repression of the widely expressed X-linked IAP (XIAP) by RNAi sensitises adult tumour cells to cytotoxics in vitro. Antisense oligonucleotide (ASO)induced down-regulation of XIAP is effective at inducing cell death and delaying the growth of adult tumour cells as xenografts and these agents are currently in phase II clinical trials. The importance of XIAP in paediatric tumours has not been characterised but high expression correlates with poor survival in childhood AML. We have used the novel XIAP ASO (AEG35156) to evaluate the effects of down-regulation of XIAP in paediatric tumour cells. Here, we show that AEG35156 can down-regulate XIAP in a number of paediatric cell lines including models of osteosarcoma, rhabdomyosarcoma and Ewing's sarcoma. Cell death assays demonstrated a higher proportion of dead cells after XIAP down-regulation by ASO and these cells displayed increased levels of cleaved caspase- 3 and cleaved PARP, showing cell death was due to apoptosis. In long-term clonogenic assays, XIAP ASO sensitised 791T osteosarcoma cells to doxorubicin, etoposide and vincristine. The work presented here suggests that AEG35156, as a monotherapy or in combination with cytotoxic agents, may be of benefit in the treatment of paediatric tumours.
\end{abstract}

Correspondence to: Dr Guy Makin, Teenage Cancer Trust Young Oncology Unit, Christie Hospital NHS Trust, Wilmslow Road, Manchester M20 4BX, UK

E-mail: guy.makin@manchester.ac.uk

Abbreviations: IAP, inhibitor of apoptosis protein; XIAP, X-linked IAP; Smac, second mitochondrial activator of apoptosis; ASO, antisense oligonucleotide

Key words: apoptosis, XIAP, paediatric cancer, cytotoxic agents

\section{Introduction}

Cancer is one of the most common causes of death amongst children between the ages of 1 and 11. Although the overall survival rate from childhood cancer is now approaching $75 \%$ (1), there still remain a subset of paediatric tumours for which survival is poor. In children presenting with metastatic osteosarcoma, metastatic Ewing's sarcoma, metastatic rhabdomyosarcoma or stage 4 neuroblastoma, survival rates remain very low despite intensive multi-agent therapy. Thus, for these diseases there is an urgent need for new agents.

Resistance to apoptotic cell death is a major survival mechanism for cancer cells and is likely to underlie the resistance to many chemotherapeutic agents (2). The apoptotic signalling cascade consists of two pathways, the intrinsic (via the mitochondria) and the extrinsic (through cell surface death receptors) leading ultimately to the activation of the cysteine-dependent aspartate specific proteases or caspases.

Inhibitor of apoptosis proteins (IAPs), prevent the activation of caspases downstream of the mitochondria. The most abundant member of this family is the X-linked IAP (XIAP). In the apoptotic cascade XIAP inhibits the cleavage of the activator caspases-3, -7 and the initiator caspase-9. Baculovirus IAP repeat (BIR) domains within the protein structure of XIAP are able to bind and inhibit caspase activation. Specifically the BIR2 domain inhibits caspase-3 and -7 and the BIR3 RING domain inhibits caspase-9 (3-5). $\mathrm{XIAP}$ is regulated endogenously by the structurally similar mitochondrial proteins SMAC and HTRA2 and by the nuclear protein XAF1. On release SMAC and HTRA2 are cleaved to active forms which bind IAPs thus preventing their association with caspases. XAF1 regulates XIAP by sequestering it to the nucleus (6).

Overexpression of XIAP in adult cancers and childhood acute myeloid leukaemia has been reported to confer chemoresistance (7). Indeed, inhibiting XIAP in several different cancer settings results in cell death and sensitises cells to chemotherapeutic agents (8-11).

AEG35156 is a second generation antisense oligonucleotide (ASO) which has been designed to target XIAP and lower the threshold of cancer cells to apoptotic stimuli whilst protecting normal healthy cells. Pre-clinically in adult tumour cell lines AEG35156, and a similar sequence AEG35146, have been reported to reduce XIAP at the mRNA 
level and induce cell death $(8,12)$. Furthermore, AEG35156 is effective as a single agent in human tumour xenograft models and enhances response to chemotherapeutic agents, including TRAIL, taxanes and vinorelbine in both in vitro and in vivo models $(8,13)$. AEG35156 is currently in an adult phase II combination study with sorafenib in first-line hepatocellular carcinoma and a phase $1 / 2$ monotherapy study in CLL/indolent B-cell lymphomas.

Here, we demonstrate that XIAP is widely expressed across a panel of paediatric tumour cell lines. In a subset of these cell lines, we show that XIAP can be down-regulated by AEG35156 and that this results in increased cell death which we suggest is due to apoptosis. Furthermore, we demonstrate XIAP down-regulation sensitises $791 \mathrm{~T}$ osteosarcoma cells to conventional chemotherapeutic agents (doxorubicin, etoposide and vincristine).

\section{Materials and methods}

Cell culture. The following cell lines were used in this study,

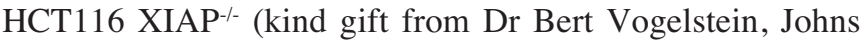
Hopkins University) $791 \mathrm{~T}$ (osteosarcoma), RD (rhabdomyosarcoma), SH-SY5Y (neuroblastoma; kindly provided by Professor Robert Ross, Fordham University, New York), RD (rhabdomyosarcoma; ECACC), CEM (acute lymphoblastic leukaemia), D283 (medulloblastoma; kindly provided by Dr Steve Clifford, University of Newcastle), RDES and A673 (Ewing's sarcoma; kindly provided by Professor Sue Burchill, CRUK Clinical Research Centre, St. James's Hospital, Leeds). Cells were maintained in RPMI-1640 or DMEM F12 media (Gibco) supplemented with $10 \%$ fetal bovine serum. All cytotoxic chemotherapeutic agents were purchased from Sigma-Aldrich ${ }^{\mathrm{TM}}$.

Antisense transfections. Cells/ml $\left(2 \times 10^{5}\right)$ were seeded per well of a 6-well plate and left to adhere for $24 \mathrm{~h}$. Stocks $(2 \mathrm{mg} / \mathrm{ml})$ of the antisense oligos against XIAP (AEG35156) and the scrambled control (AEG35187) were formulated in sterile water. Transfection of the oligos was facilitated by Oligofectamine (Invitrogen) in OptiMEM media as per manufacturer's instructions. Prior to the addition of transfection reagents, cells were washed twice and growth media replaced with OptiMEM media. Transfection reagents were added to the cells and left to incubate for $16 \mathrm{~h}$, after which time cells were washed twice with PBS and fresh growth media added.

Immunoblotting. Protein lysates were prepared as previously described (14) resolved by sodium dodecyl sulphate polyacrylamide gel electrophoresis and transferred to PVDF membrane (Immobilon). Standard immunoblotting procedures were followed with overnight incubation at $4{ }^{\circ} \mathrm{C}$ with the following primary antibodies: XIAP 1:1000 (Clone 28, BD Transduction Laboratories), cIAP-1 and cIAP-2 1:1000 (R\&D Systems), Survivin $1 \mu \mathrm{g} / \mathrm{ml}$ (Novus Biologicals), SMAC 1:1000 (BD Transduction Laboratories), XAF1 1:1000 (Imgenex), PARP 1:1000 (Cell Signalling), cleaved caspase-3 1:100 (Cell Signalling), Tubulin 1:1000 (Cell Signaling) and GAPDH 1:2000 (Abcam). Blots were visualised with the enhanced chemiluminescence system (Amersham) and analyzed on a Fuji LAS-1000 Plus imaging system with AIDA software (Fuji).
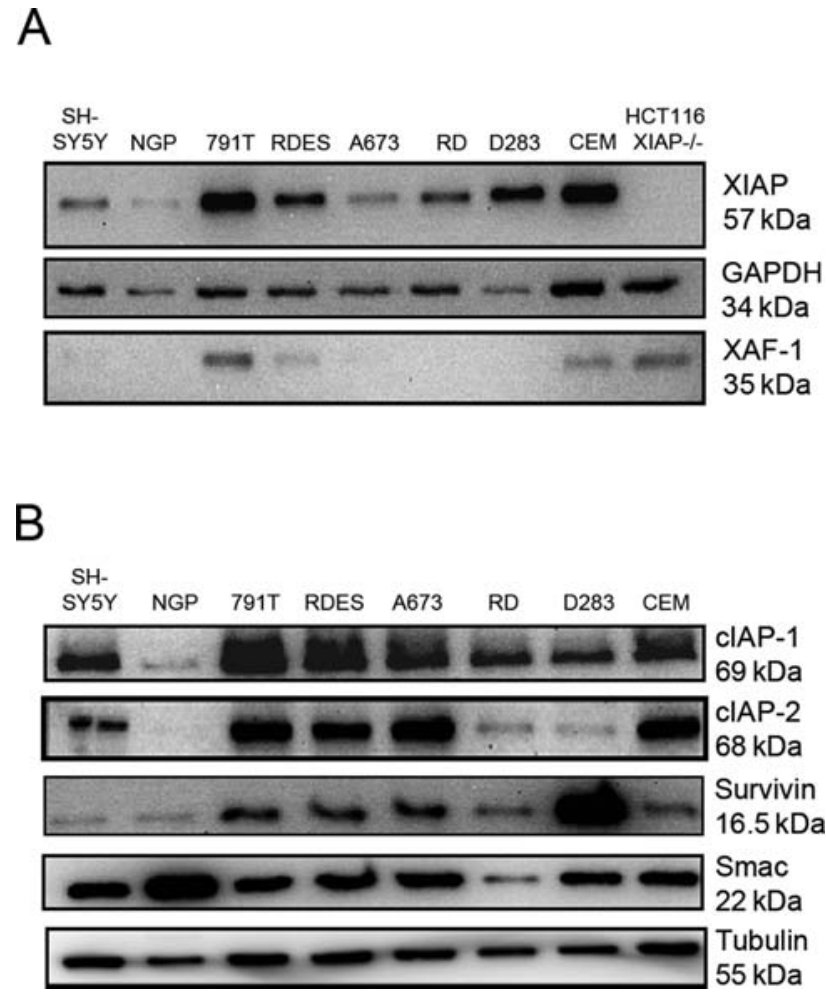

Figure 1. Immunoblot analysis across a panel of paediatric tumour cell lines showing; (A) XIAP, Xaf-1 and (B) cIAP1, cIAP2, survivin and Smac protein expression levels. The human tumour cell line HCT116 $\mathrm{XIAP}^{-/-}$is shown as a negative control for XIAP expression. GAPDH and tubulin were used as protein loading controls

Live/dead counts. At 24, 48 and 72 h post-transfection, scrambled and XIAP antisense transfected cells were harvested and immediately analysed for live/dead analysis by the incorporation of trypan blue. Three counts were performed per sample on a haemocytometer and the average live or dead cells were recorded. Each experiment was performed 3 times on separate occasions and results presented as an average of the 3 studies \pm SEM.

Clonogenic assays. Twenty-four hours post transfection of ASO, 500 cells per well were plated into 6-well plates. Fortyeight hours post-transfection cells were treated with varying concentrations of either, cisplatin, doxorubicin, etoposide or vincristine for $1 \mathrm{~h}$, before the medium was aspirated, cells washed with PBS, and fresh medium added. Plates were incubated at $37^{\circ} \mathrm{C}$ and $5 \% \mathrm{CO}_{2}$ for $10-14$ days until visible colonies ( $>50$ cells) were present in the untreated well. All assays were performed in triplicate. The surviving fraction was calculated as number of colonies in the test condition/number of colonies in the untreated well and plotted logarithmically against drug concentration.

\section{Results}

XIAP is widely expressed across a panel of paediatric tumour cell lines. XIAP expression in paediatric tumour cell lines has been previously reported in acute lymphoblastic leukaemia (ALL) (15) and in childhood AML (7). However, we wanted to investigate the therapeutic potential of down-regulating 
A



B

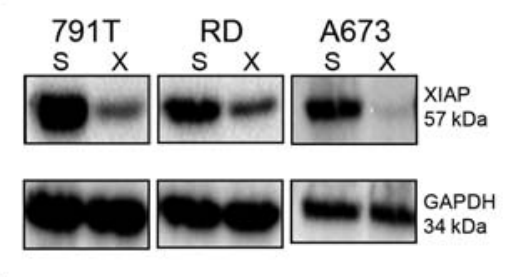

C



Figure 2. AEG35156 down-regulates XIAP (A) maximally at $48 \mathrm{~h}$ post transfection (B) across a panel of paediatric tumour cell lines including 791T (osteosarcoma), A673 (Ewing's sarcoma) and RD (rhabdomyosarcoma) and (C) does not alter expression of cIAP1 or survivin in 791T or RD cells. GAPDH was used as a loading control on the immunoblots. S, scrambled ASO (AEG35187); X, XIAP ASO (AEG35156).

XIAP across a panel of paediatric tumour cell lines. We have previously screened 29 paediatric tumour cell lines and observed XIAP expression in a large proportion of cells. Here, we show a panel of paediatric tumour cell lines, from a range of disease settings [CEM (leukaemia), D283 (medulloblastoma), RD (rhabdomyosarcoma), NGP and SHSY5Y (neuroblastoma), 791T (osteosarcoma) and A673 and RDES (Ewing's sarcoma)], with similar expression levels of XIAP to the adult colorectal cancer cell line HCT116, which expresses XIAP at a level above the mean for the NCI60 panel (Fig. 1A). Across this panel we observed only low levels of expression of the endogenous XIAP inhibitor XAF1.

Expression of other IAP family members, cIAP1 and cIAP2, in these cell lines was variable but correlated such that cells expressing lower levels of cIAP-1, such as the MYCN amplified neuroblastoma cell line NGP, the rhabdomyosarcoma cell line RD and the medulloblastoma cell line D283, also expressed low levels of cIAP-2 (Fig. 1B). Expression of survivin, another member of the IAP family, was also variable with highest expression in D283 cells, and relatively low level expression in SH-SY5Y and NGP neuroblastoma cells. Smac, a protein known to relieve the inhibitory effect of a number of IAPs, was expressed at a similar level across the panel of cell lines except for RD cells, with lower level expression, and NGP cells with higher level expression.

AEG35156 antisense down-regulates XIAP expression in paediatric cancer cell lines. We selected three cell lines from different types of paediatric cancer (791T, A673 and RD) which expressed XIAP (as well as cIAP-1, cIAP-2, survivin
A

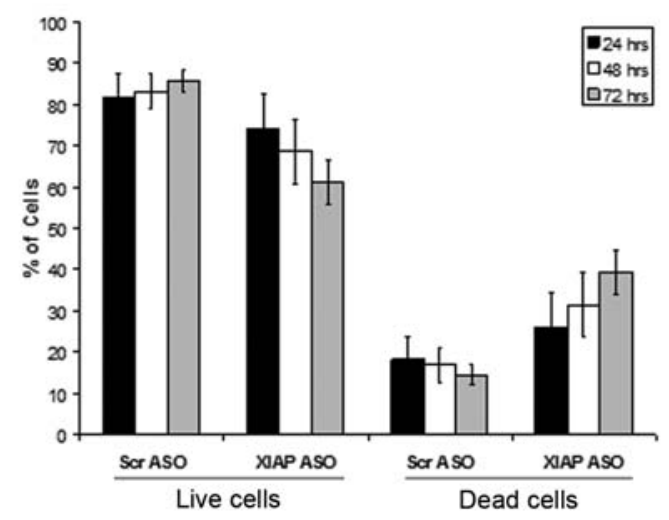

B

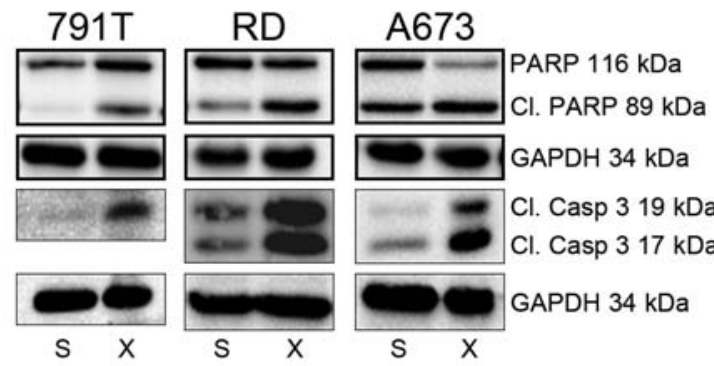

Figure 3. Down-regulation of XIAP in 791 T cells by AEG35156 induces (A) cell death as measured by a trypan blue live/dead assay, and results in (B) the cleavage of PARP and caspase-3 in 791T, A673 and RD cells. All experiments are representative of 3 individual experiments and error bars are \pm SEM. S, scrambled ASO (AEG35187); X, XIAP ASO (AEG35156).

and Smac) at variable levels (Fig. 1A). In order to determine the optimum time for XIAP down-regulation, the 791T cell line was transfected with either the XIAP or scrambled ASO and cells harvested $24,48,72$ or 96 h post transfection. Immunoblot analysis of XIAP protein levels suggested that XIAP repression was maximal $48 \mathrm{~h}$ post-transfection and that protein levels were starting to recover beyond $72 \mathrm{~h}$ (Fig. 2A). Immunoblot analysis demonstrated that protein levels of XIAP were decreased $48 \mathrm{~h}$ after transfection of XIAP ASO in all three cell lines (Fig. 2B).

To ensure that the XIAP antisense was not inducing compensatory changes in other IAP family members, 791T and RD cells were analysed for cIAP1 and survivin levels after transfection. In both cell lines cIAP1 and survivin levels did not significantly change after XIAP down-regulation compared to controls (Fig. 2C).

Down-regulation of XIAP enhances cell death and induces apoptosis in paediatric cancer cells. In order to investigate if XIAP repression induced cell death, $791 \mathrm{~T}$ cells were transfected with XIAP or control antisense and samples harvested 24,48 or $72 \mathrm{~h}$ post-transfection. At these time points cell death was determined by counting live/dead cells by trypan blue exclusion. In the scrambled control group, the number of live and dead cells was consistent across the time course at $\sim 80$ and $\sim 15 \%$ of cells respectively (Fig. 3A). In contrast, 
A

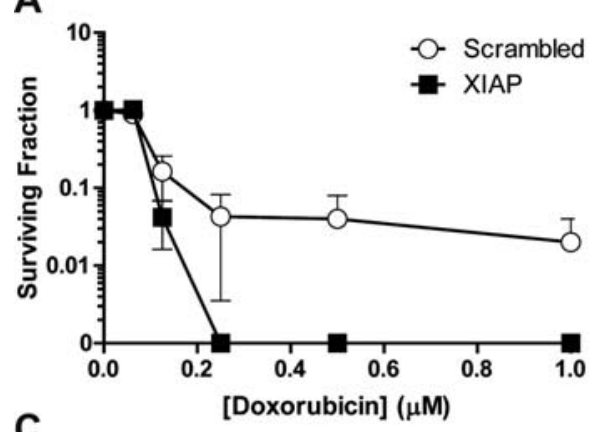

C

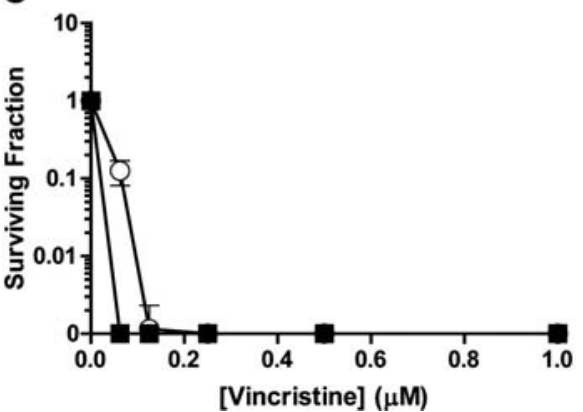

B
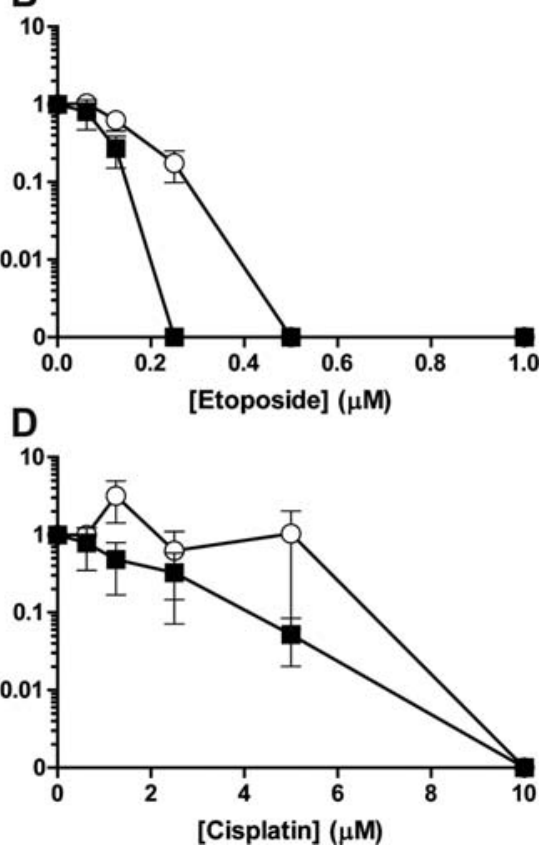

Figure 4. Surviving fraction of cells from a clonogenic assay post-transfection with control scrambled ASO (open circles) or XIAP (AEG35156) ASO (closed squares) which $48 \mathrm{~h}$ post transfection were exposed to chemotherapeutic agents; (A) doxorubicin, (B) etoposide, (C) vincristine or (D) cisplatin. Results are presented as the average of 3 independent experiments and error bars are \pm SEM.

when XIAP was down-regulated the number of dead cells $24 \mathrm{~h}$ post-transfection was initially higher $(\sim 25 \%)$ compared to the control group $(\sim 15 \%)$ (Fig. 3A). The number of dead cells increased over time in the XIAP antisense treatment and after $72 \mathrm{~h} \sim 40 \%$ of cells were dead compared to $\sim 15 \%$ in the control group. In order to determine if the cell death observed was a result of apoptosis, 791T, A673 and RD cells were transfected with control or XIAP antisense, harvested $48 \mathrm{~h}$ post transfection and analysed for cleavage of caspase- 3 and PARP. In all three cell lines PARP and caspase- 3 cleavage was observed after XIAP down-regulation (Fig. 3B). These findings suggest that down-regulating XIAP in paediatric cancer cells results in an increase in apoptotic cell death.

AEG35156 sensitises $791 T$ cells to cytotoxic agents in a clonogenic assay. We finally wanted to know if AEG35156 could sensitise paediatric tumour cells to clinically relevant chemotherapeutic agents and enhance the cytotoxic effect of these agents. Twenty-four hours post-transfection of the control or XIAP antisense, 791T cells were harvested and plated out for clonogenic assays followed, $24 \mathrm{~h}$ later by a $1 \mathrm{~h}$ treatment with varying concentrations of either cisplatin, doxorubicin, etoposide or vincristine. Cells were then left for approximately 14 days to allow colonies to establish. A $1 \mathrm{~h}$ exposure of AEG35156-transfected cells to doxorubicin (Fig. 4A), etoposide (Fig. 4B) or vincristine (Fig. 4C), induced a marked decline in clonogenic survival compared to control transfected cells. In contrast exposure of XIAP down-regulated cells to cisplatin (Fig. 4D), did not appear to have an effect upon clonogenic survival compared to control populations. The observations here suggest that down-regulation of XIAP with AEG35156 sensitises 791T osteosarcoma cells to doxorubicin, etoposide and vincristine, but not cisplatin.

\section{Discussion}

In this report we show that XIAP can be down-regulated in a range of paediatric tumour cell lines by the antisense oligonucleotide, AEG35156. Detailed analysis of the osteosarcoma cell line 791T, showed that XIAP down-regulation induced cell death which bore the hallmarks of apoptosis. Furthermore, XIAP repression sensitised $791 \mathrm{~T}$ cells to conventional cytotoxic agents in a clonogenic assay.

In adult cancer, members of the IAP family are upregulated in a wide range of tumour types and this confer a poor clinical outcome (16). In paediatric cancers, high expression levels of the IAP family members, in particularly XIAP, have been linked with poor clinical outcomes in medulloblastoma, neuroblastoma and AML (17-19). Therefore, down-regulating XIAP in paediatric tumours may offer a novel therapeutic strategy for childhood cancer. Transient transfection of the XIAP antisense, AEG35156, lead to down-regulation of XIAP across a panel of paediatric tumour cell lines; 791T (osteosarcoma), A673 (Ewing's sarcoma) and RD (rhabdomyosarcoma), without altering levels of other IAP proteins, consistent with observations in adult tumour cell lines $(12,13)$.

XIAP down-regulation alone was sufficient to induce cell death in 791T osteosarcoma cells, and this was due to an increase in apoptosis. In addition, previous observations from our laboratory, using a small molecule inhibitor of XIAP, have demonstrated induction of apoptosis in paediatric tumour cell lines (unpublished data). Indeed, down-regulation/inhibition of XIAP alone in childhood ALL, and in several adult tumour types, has been shown to be sufficient to enhance cell death $(12,15,20,21)$. However, not all tumour cells readily undergo apoptosis in response to XIAP inhibition. It is likely that 
there are subtle differences in the thresholds between cell lines which prime them to undergo apoptosis in response to XIAP inhibition/down-regulation.

Despite the activity of XIAP ASO as a single agent, it is likely that it will be used in combination with conventional cytotoxic agents in the clinic. Cancer cell lines which express high levels of XIAP are resistant to conventional chemotherapeutic agents and can be sensitised by co-treatment with XIAP ASO (12). In $791 \mathrm{~T}$ osteosarcoma cells combining XIAP ASO with doxorubicin, etoposide or vincristine, resulted in greater sensitivity to the cytotoxic agent in a clonogenic assay. However, down-regulation of XIAP did not sensitise to cisplatin, an interaction that we have also previously observed with a small molecule inhibitor of XIAP (unpublished data). However, in the combination studies we describe here cells were exposed to cisplatin $48 \mathrm{~h}$ following transfection with XIAP ASO, as this was the time point at which we saw the greatest down-regulation of XIAP protein levels. It is possible that with different scheduling between XIAP ASO and cisplatin dosing, a different interaction may emerge. In these combination experiments $791 \mathrm{~T}$ cells were only treated once with XIAP antisense; it is possible that multiple transfections of XIAP ASO may be needed to continually suppress XIAP mRNA and protein turnover in order for sensitisation to cisplatin to occur (13).

The effectiveness of XIAP ASO at inducing apoptosis and sensitising to conventional cytotoxic agents in paediatric solid tumour cell lines, in conjunction with the similar effects seen in paediatric ALL (15), suggest that XIAP inhibition may be a useful novel therapeutic strategy in drug-resistant paediatric cancer.

\section{Acknowledgements}

This study was supported by Cancer Research UK (Grant code C8232) and NIH (CA-113318).

\section{References}

1. Gatta G, Capocaccia R, Stiller C, et al: Childhood cancer survival trends in Europe: a EUROCARE working group study. J Clin Oncol 23: 3742-3751, 2005.

2. Hanahan D and Weinberg RA: The hallmarks of cancer. Cell 100: 57-70, 2000

3. Chai J, Wu Q, Shiozaki E, Srinivasula SM, Alnemri ES and Shi Y: Crystal structure of a procaspase-7 zymogen: mechanisms of activation and substrate binding. Cell 107: 399-407, 2001.

4. Riedl SJ, Renatus M, Schwarzenbacher R, et al: Structural basis for the inhibition of caspase-3 by XIAP. Cell 104: 791-800, 2001.
5. Shiozaki EN, Chai J, Rigotti DJ, et al: Mechanism of XIAPmediated inhibition of caspase-9. Mol Cell 11: 519-527, 2003.

6. Liston P, Fong WG, Kelly NL, et al: Identification of XAF1 as an antagonist of XIAP anti-caspase activity. Nat Cell Biol 3: 128-133, 2001.

7. Tamm I, Richter S, Scholz F, et al: XIAP expression correlates with monocytic differentiation in adult de novo AML: impact on prognosis. Hematol J 5: 489-495, 2004.

8. Hu Y, Cherton-Horvat G, Dragowska V, et al: Antisense oligonucleotides targeting XIAP induce apoptosis and enhance chemotherapeutic activity against human lung cancer cells in vitro and in vivo. Clin Cancer Res 9: 2826-2836, 2003.

9. Vogler M, Walczak H, Stadel D, et al: Small molecule XIAP inhibitors enhance TRAIL-induced apoptosis and antitumor activity in preclinical models of pancreatic carcinoma. Cancer Res 69: 2425-2434, 2009

10. Shaw TJ, Lacasse EC, Durkin JP and Vanderhyden BC: Downregulation of XIAP expression in ovarian cancer cells induces cell death in vitro and in vivo. Int J Cancer 122: 1430-1434, 2008.

11. Loeder S, Drensek A, Jeremias I, Debatin KM and Fulda S: Small molecule XIAP inhibitors sensitize childhood acute leukemia cells for CD95-induced apoptosis. Int J Cancer 126: 2216-2228, 2010

12. McManus DC, Lefebvre CA, Cherton-Horvat G, et al: Loss of XIAP protein expression by RNAi and antisense approaches sensitizes cancer cells to functionally diverse chemotherapeutics. Oncogene 23: 8105-8117, 2004.

13. LaCasse EC, Cherton-Horvat GG, Hewitt KE, et al: Preclinical characterization of AEG35156/GEM 640, a second-generation antisense oligonucleotide targeting X-linked inhibitor of apoptosis. Clin Cancer Res 12: 5231-5241, 2006.

14. Hussein D, Estlin EJ, Dive C and Makin GW: Chronic hypoxia promotes hypoxia-inducible factor-1alpha-dependent resistance to etoposide and vincristine in neuroblastoma cells. Mol Cancer Ther 5: 2241-2250, 2006.

15. Fakler M, Loeder S, Vogler M, et al: Small molecule XIAP inhibitors cooperate with TRAIL to induce apoptosis in childhood acute leukemia cells and overcome bcl-2-mediated resistance. Blood 113: 1710-1722, 2009.

16. Tamm I, Kornblau SM, Segall H, et al: Expression and prognostic significance of IAP-family genes in human cancers and myeloid leukemias. Clin Cancer Res 6: 1796-1803, 2000.

17. Haberler C, Slave I, Czech T, et al: Histopathological prognostic factors in medulloblastoma: high expression of survivin is related to unfavourable outcome. Eur J Cancer 42: 2996-3003, 2006.

18. Potts PR, Singh S, Knezek M, Thompson CB and Deshmukh M: Critical function of endogenous XIAP in regulating caspase activation during sympathetic neuronal apoptosis. J Cell Biol 163: 789-799, 2003

19. Dasgupta A, Alvarado CS, Xu Z and Findley HW: Expression and functional role of inhibitor-of-apoptosis protein livin (BIRC7) in neuroblastoma. Biochem Biophys Res Commun 400: 53-59, 2010.

20. Schimmer AD, Welsh K, Pinilla C, et al: Small-molecule antagonists of apoptosis suppressor XIAP exhibit broad antitumor activity. Cancer Cell 5: 25-35, 2004

21. Wang Z, Cuddy M, Samuel T, et al: Cellular, biochemical, and genetic analysis of mechanism of small molecule IAP inhibitors. J Biol Chem 279: 48168-48176, 2004 